\title{
Extracción de taninos de cortezas de tres especies árboles de la región de la Orinoquia
}

\section{Extraction of bark tannins from three tree species in the Orinoquia region}

\section{Extração de taninos de casca de três espécies arbóreas da região de}

\section{Orinoquia}

Carreño Morales Alba Milena ${ }^{1}$ y Jiménez Forero Javier Alexander ${ }^{2}$ ${ }^{1}$ Ingeniera Agroindustrial, Universidad de los Llanos y ${ }^{2}$ Ingeniero de Producción Agroindustrial, MSc. Docente de la Universidad de los Llanos

jajimenez@unillanos.edu.co

Recibido 25 de Mayo 2018, Aceptado 22 de Octubre 2018

\section{RESUMEN}

La Orinoquia se caracteriza por su gran riqueza forestal nativa y comercial, a través del tiempo se han desarrollado investigaciones con el fin de fomentar su desarrollo y los diferentes usos de las especies vegetales. En esta investigación se evaluó la extracción de taninos a partir de la corteza de árboles que se encuentran en la región de la Orinoquia en Colombia, las especies evaluadas fueron pino (Pinus caribaea), acacia (Acacia mangium) y eucalipto (Eucalypto pellita). Se realizó una selección de las cortezas de los árboles mencionados para secarlas y luego molerlas, y así poder reducir sus partículas hasta un tamaño de $1 \mathrm{~mm}$, se llevó a cabo una operación de lixiviación solido-líquido para evaluar los siguientes factores: concentración de $\mathrm{NaOH}$ : 1 y $5 \%$, relación solido-líquido (S/L): 1/60, 1/90 y 1/120, y temperatura de extracción: 70 y $80^{\circ} \mathrm{C}$, observándose el efecto sobre el porcentaje de rendimiento de extracción de taninos. Se realizó un análisis estadístico en el programa R-Project, mediante el test de Fisher con un 95\% de confiabilidad. Se obtuvo como resultado que la corteza con mayor producción fue la de pino en condiciones de $5 \% \mathrm{NaOH}$, relación $S / L$ de $1 / 90$ y temperatura de $80^{\circ} \mathrm{C}$, en eucalipto se obtuvieron mejores rendimientos con $5 \% \mathrm{NaOH}, 1 / 120$ y $80^{\circ} \mathrm{C}$, por último, en acacia el mejor comportamiento se observó con $1 \% \mathrm{NaOH}, 1 / 90$ y $80^{\circ} \mathrm{C}(\mathrm{P}<0.05)$. Se concluye que la temperatura no influye directamente en el rendimiento de la extracción, puesto 
que la diferencia entre los dos niveles no fue importante, caso contrario se observó con los factores de alcalinidad y la relación S/L, obteniéndose mayores rendimientos con $5 \%$ Vs $1 \%$ de $\mathrm{NaOH}$ y una relación ente soluto solvente $1 / 90$ Vs $1 / 60$ y 1/120.

Palabras clave: Metabolitos secundarios, polímeros, toxinas, lixiviación.

\begin{abstract}
The Orinoquia is characterized by its great native and commercial forest wealth, throughout time, research has been developed in order to promote their development and the different uses of plant species. In this research, the extraction of tannins from the bark of trees found in the Orinoquia region of Colombia was evaluated. The evaluated species were pine (Pinus caribaea), acacia (Acacia mangium) and eucalyptus (Eucalypto pellita). A selection of the barks of the mentioned trees was made to dry them and then grind them, and thus be able to reduce its particles to a size of $1 \mathrm{~mm}$, a solid-liquid leaching operation was carried out to evaluate the following factors: $\mathrm{NaOH}$ concentration: 1 and $5 \%$, solid-liquid ratio $(\mathrm{S} / \mathrm{L}): 1 / 60,1 / 90$ and $1 / 120$, and extraction temperature: 70 and $80^{\circ} \mathrm{C}$, observing the effect on the percentage of extraction yield of tannins. A statistical analysis was carried out in the R-Project program, using the Fisher test with 95\% reliability. It was obtained as a result that the bark with the highest production was pine under conditions of $5 \% \mathrm{NaOH}, \mathrm{S} / \mathrm{L}$ ratio of $1 / 90$ and temperature of $80^{\circ} \mathrm{C}$, in eucalyptus, better yields were obtained with $5 \% \mathrm{NaOH}, 1 / 120$ and $80^{\circ} \mathrm{C}$, finally, in acacia the best performance was observed with $1 \% \mathrm{NaOH}, 1 / 90$ and $80^{\circ} \mathrm{C}(\mathrm{P}<0.05)$. It is concluded that the temperature does not directly influence the extraction performance, since the difference between the two levels was not important, otherwise it was observed with the alkalinity factors and the S/L ratio, obtaining higher yields with $5 \% \mathrm{Vs} 1 \% \mathrm{NaOH}$ and a solvent solute ratio $1 / 90 \mathrm{Vs} 1 / 60$ and $1 / 120$.
\end{abstract}

Keywords: Secondary metabolites, polymers, toxins, leaching. 


\section{RESUMO}

A Orinoquia é caracterizada por sua grande riqueza florestal nativa e comercial, ao longo do tempo, pesquisas foram desenvolvidas com o objetivo de promover o seu desenvolvimento e os diferentes usos das espécies vegetais. Nesta pesquisa, avaliou-se a extração de taninos da casca das árvores encontradas na região de Orinoquia, na Colômbia. As espécies avaliadas foram pinus (Pinus caribaea), acácia (Acacia mangium) e eucalipto (Eucalypto pellita). Uma seleção das cascas das árvores mencionadas foi feita para secá-las e depois moê-las, e assim poder reduzir suas partículas a um tamanho de $1 \mathrm{~mm}$, uma operação de lixiviação sólido-líquido foi realizada para avaliar os seguintes fatores: Concentração de $\mathrm{NaOH}: 1$ e 5\%, relação sólido-líquido (S/L): 1/60, 1/90 e 1/120, e temperatura de extração: 70 e $80^{\circ} \mathrm{C}$, observando o efeito na porcentagem de rendimento de extração de taninos. Uma análise estatística foi realizada no programa R-Project, utilizando o teste de Fisher com 95\% de confiabilidade. Obteve-se como resultado que a casca com maior produção era pinheiro sob condições de $5 \% \mathrm{NaOH}$, relação $\mathrm{S} / \mathrm{L}$ de $1 / 90 \mathrm{e}$ temperatura de $80^{\circ} \mathrm{C}$, em eucalipto, melhores rendimentos foram obtidos com $\mathrm{NaOH} 5 \%, 1 / 120$ e $80^{\circ} \mathrm{C}$, finalmente, em acácia, o melhor desempenho foi observado com $\mathrm{NaOH}$ a $1 \%, 1 / 90$ e $80^{\circ} \mathrm{C}(\mathrm{P}<0.05)$. Conclui-se que a temperatura não influencia diretamente no desempenho da extração, já que a diferença entre os dois níveis não foi importante, caso contrário foi observado com os fatores de alcalinidade e a relação $S / L$, obtendo rendimentos mais elevados com $5 \%$ Vs $1 \%$ $\mathrm{NaOH}$ e uma razão solvente soluto 1/90 Vs 1/60 e 1/120.

Palavras-chave: Metabolitos secundários, polímeros, toxinas, lixiviação.

\section{INTRODUCCIÓN}

En Colombia 17 millones de hectáreas son aptas para reforestación distribuidas en varios pisos altitudinales, actualmente sólo se está usando el $2.06 \%$ del potencial de las plantaciones forestales en 350.000 hectáreas. La Orinoquia se caracteriza por su gran riqueza forestal nativa y comercial, a través del tiempo se han desarrollado investigaciones con el fin de fomentar su desarrollo y los diferentes 
usos de la madera, siendo esta zona propicia para establecer un renglón agroindustrial fuerte para la transformación u obtención de productos a partir de esta materia prima (PROEXPORT, 2012).

El eucalipto (Eucalipto pellita) es una variedad comercial, su madera y subproductos son muy utilizados en la región, tiene la cualidad de contener polifenoles en niveles superiores al $30.6 \%$, lo cual despierta interés como fuente de taninos objeto de esta investigación (Borralho y Nieto, 2012). Otra especie de interés es el pino caribe (Pinus caribaea), plantación que es de transcendencia en el mundo por su madera fuerte y manejable para la elaboración de muebles y otros objetos, siendo su corteza un residuo de la transformación de la madera sin uso eficiente que ha generado un problema de contaminación debido al gran volumen de comercialización de esta especie (Villanueva et al., 2015). La corteza de pino es rica en compuestos fenólicos (Jerez et al., 2006), en algunos estudios realizados han encontrado taninos como catequina, epicatequina, epigalocatequina y galato de epicatequina (Jahanshahi et al., 2016). Los extractos de la corteza del pino tienen propiedades anti-radicales, anti-inflamatorios y antioxidantes (Amoako y Awika, 2016).

La Acacia mangium otra de las especies usadas en la investigación, crece rápidamente y se adapta con facilidad a las diferentes condiciones que pueden ofrecer los suelos, por tal motivo se ha convertido en una especie en desarrollo para mantener la sostenibilidad de la madera en el mundo (Nadhari et al., 2014). La corteza es un material de composición química más compleja que la de la madera, distinguiéndose sobre todo por el alto contenido de sustancias extraíbles, principalmente polifenoles y suberina; entre los polifenoles se encuentran los fenoles simples, lignanos, estilbenos, flavonoides, quinonas y taninos (Álvarez, 2007).

Las cortezas de los mencionado árboles son importante fuente de polifenoles, los cuales son un grupo extenso de sustancias no energéticas presentes en diferentes estructuras de origen vegetal (Quiñones et al., 2012); estos compuestos tienen propiedades antioxidantes que las hacen sustancias de interés para diferentes áreas entre esas la de salud y nutrición (Casares, 2010; Coronado et al., 2015). 
Entre esta gran variedad de sustancias se encuentran los taninos, los cuales según su definición química son metabolitos secundarios derivados de plantas que pueden ser esteres de ácido gálico o sus derivados, unidos a una amplia variedad de polioles, catequinas u oligomeros de proantocianidinas (Olivas et al., 2015).

Los taninos se dividen en dos tipos: condensados e hidrolizables, y se encuentran como metabolitos secundarios en diferentes especies vegetales, dentro de todos los polifenoles son considerados como los de mayor valor económico siendo su principal uso como curtiente de pieles de animales (Colín et al., 2013), puesto que cuentan con una cualidad sensorial de generar sabor amargo, astringencia y estabilidad de color, factores importantes para generar características deseables en los vinos (Durán, 2010), por lo tanto las uvas, materia prima de los vinos, son ricas en taninos.

La extracción química es la separación de un componente especifico, en este caso los taninos, proceso que se inicia con una molienda formando astillas o virutas; existen varios métodos dependiendo de la parte de la planta utilizada, los cuales pueden darse de manera empírica e industrial, que puede realizarse por difusión a tanque abierto (Wadnerkar et al., 2016), colado, autoclave, contracorriente y por último el que fue utilizado es esta investigación el de lixiviación (Casares, 2010), el cual puede realizarse por medio alcohólico u alcalino (Naima et al., 2015). El objetivo de este trabajo fue aplicar diferentes tratamientos con factores o condiciones, realizando combinaciones de los medios y obteniendo así una respuesta con cada una de las especies de árboles.

\section{METODOLOGÍA}

\section{Obtención y acondicionamiento de las muestras}

Se realizó la obtención del material vegetal en una empresa productora de maderas y sus subproductos ubicada en Villavicencio, Meta, Colombia (Figura 1), se colectaron cortezas correspondientes a las variedades de acacia (Acacia mangium), eucalipto (Eucalipto pellita) y pino (Pinus caribaea). Se realizó el acondicionamiento de las cortezas, reduciendo su tamaño en dos equipos de molienda; inicialmente se 
usó un molino de granos con tamices que permitieron dejarlas en partículas más pequeñas, para luego ser procesadas en un molino analítico, logrando obtener un tamaño de un $1 \mathrm{~mm}$, empleando tamices №. 20 en la serie de Tyler (Chupin et al., 2013) (Anexo 2). Como última parte del acondicionamiento de las muestras se realizó un secado en una estufa de aire forzado a temperatura de $40^{\circ} \mathrm{C}$, evitando las pérdidas por volatilización de los taninos (Figura 1).
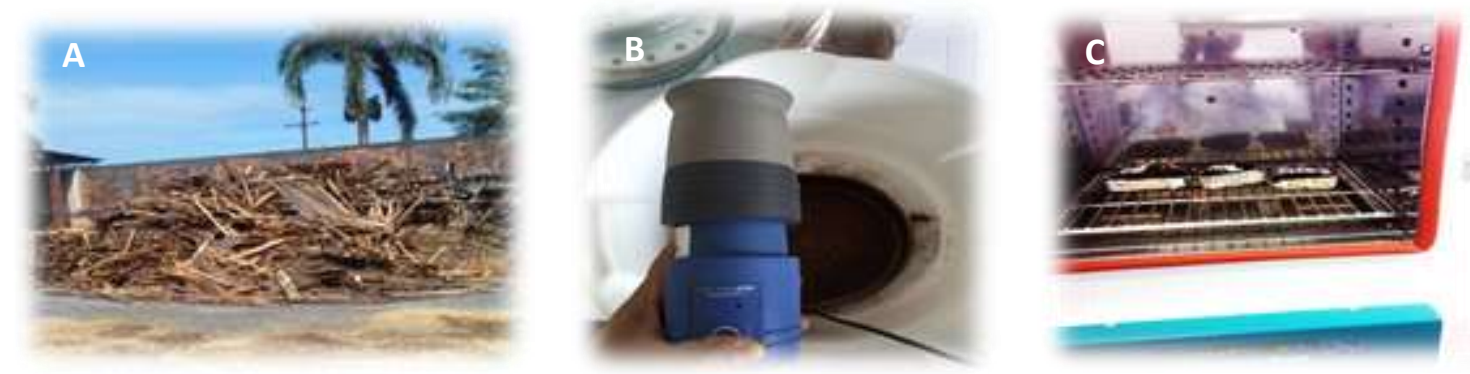

Figura 1. A. Cortezas del sector forestal, B. Molino analítico de laboratorio. C. Horno de secado

\section{Extracción}

Se realizó un proceso de lixiviación solido-líquido con solventes, bajo un diseño de 12 tratamientos con tres replicas cada uno, teniendo en cuenta los siguientes tres factores: relación solido-líquido S/L: 1/60, $1 / 90$ y 1/120; concentración de $\mathrm{NaOH}$ con dos niveles de 1 y $5 \%$; y como tercer factor dos niveles de temperatura 70 y $80^{\circ} \mathrm{C}$, para un total de 36 unidades experimentales (Tabla 1). Se realizó un análisis estadístico en el programa R-Project, mediante el test de análisis estadístico Fisher con un $95 \%$ de confiabilidad, obteniendo resultados de rendimiento según los factores involucrados.

Todos los tratamientos se disolvieron en $50 \mathrm{ml}$ de agua destilada, además de estos solventes se adicionó de manera estándar sulfito de sodio $\left(\mathrm{Na}_{2} \mathrm{SO}_{3}\right) 0.125 \mathrm{~g}$ y sulfito de sodio hidrogenado $\left(\mathrm{Na}_{2} \mathrm{HSO}_{3}\right) 0.32 \mathrm{ml}$ que sirvieron de coadyuvantes para la extracción. 
Tabla 1. Componentes y tratamientos para la extracción de taninos

\begin{tabular}{cccccc}
\hline Tratamiento & $\mathbf{T}^{\circ}\left({ }^{\circ} \mathbf{C}\right)$ & NaOH (gr) & $\begin{array}{c}\text { Extracto } \\
\text { (gr) }\end{array}$ & $\begin{array}{c}\text { Relación } \\
\mathbf{S} / \mathbf{L}\end{array}$ & Árbol \\
\hline 1 & 80 & 0.5 & 0.83 & 1 a 60 & Acacia \\
2 & 80 & 0.5 & 0.55 & 1 a 90 & Eucalipto \\
3 & 80 & 0.5 & 0.4 & 1 a 120 & Pino \\
4 & 80 & 0.25 & 0.83 & 1 a 60 & Acacia \\
5 & 80 & 0.25 & 0.55 & 1 a 90 & Eucalipto \\
6 & 80 & 0.25 & 0.4 & 1 a 120 & Pino \\
7 & 70 & 0.5 & 0.83 & 1 a 60 & Acacia \\
8 & 70 & 0.5 & 0.55 & 1 a 90 & Eucalipto \\
9 & 70 & 0.5 & 0.4 & 1 a 120 & Pino \\
10 & 70 & 0.25 & 0.83 & 1 a 60 & Acacia \\
11 & 70 & 0.25 & 0.55 & 1 a 90 & Eucalipto \\
12 & 70 & 0.25 & 0.4 & 1 a 120 & Pino \\
\hline
\end{tabular}

Cada uno de los tratamientos se disolvieron en $50 \mathrm{ml}$ de agua y se les adicionaron: $0.125 \mathrm{de}$ $\mathrm{Na}_{2} \mathrm{SO}_{3}$ y $0.32 \mathrm{ml}$ de $\mathrm{Na}_{2} \mathrm{HSO}_{3}$. $\mathrm{T}^{\circ}=$ Temperatura de extracción. $\mathrm{S} / \mathrm{L}=$ Solido-liquido. Concentración de $\mathrm{NaOH} 1 \%(0.5 \mathrm{~g})$ y $5 \%(0.25 \mathrm{~g})$.

A cada una de los tratamientos ya mezclados se les aplicó un calentamiento en plancha con agitación constante por un periodo de 120 minutos, terminando este proceso, se hizo un filtrado del sobrenadante utilizando papel whattman \#1 y el residuo se lavó con agua, los filtrados se secaron a $50^{\circ} \mathrm{C}$, obteniendo la muestra seca correspondiente al extracto recuperado, para conocer el rendimiento se empleó la ecuación propuesta por Granados et al., (2014):

$$
\% \text { Rendimiento extracción }=\frac{\text { Masa recuperada }}{\text { Masa inicial }} \times 100
$$

\section{RESULTADOS Y DISCUSIÓN}

El porcentaje de rendimiento de extracción de taninos fue menor con la corteza de eucalipto con un promedio de $48 \%$ (Gráfica 1), estos resultados concuerdan con otras investigaciones en las cuales se confirma que los compuestos fenólicos como los taninos tienen una descomposición rápida al momento de realizarse la 
extracción, puesto que son antioxidantes su cantidad se reduce drásticamente (Min et al., 2015). El rendimiento de pino fue mayor en $0.7 \%$ con respecto a la acacia, generando una extracción de taninos de $0.69 \%$, al respecto Aguilar et al., (2012) coinciden en que la corteza con mayor rendimiento de extracción es la de pino; es importante resaltar que la misma está condicionada a la hidrólisis, tipo y concentraciones de los reactivos utilizados en los materiales lignocelulósicos como las cortezas de árboles, pues la rotura de la hemicelulosa es selectiva para liberar los taninos y en ocasiones conduce a la producción de otros compuestos bajando la producción de estos fenoles (Garrote et al., 2007).

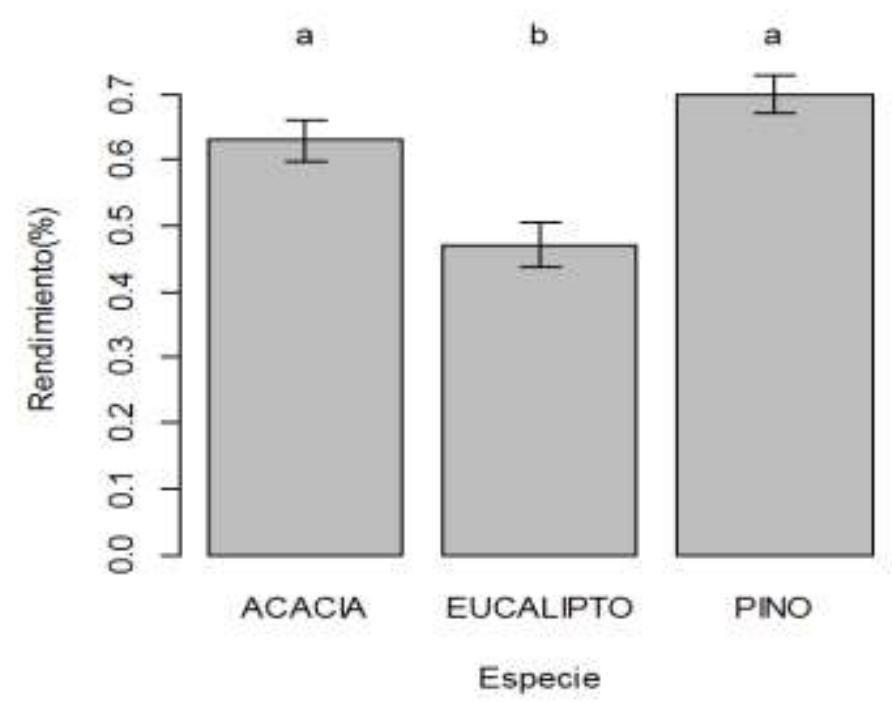

Promedios con letra distinta indica significativa según la prueba de Fisher $(P<0.05)$

Gráfica 1. Porcentaje (\%) de rendimiento de extracción de taninos en las tres especies de árboles

Se observó que durante el proceso de extracción las temperaturas evaluadas no presentaron una diferencia estadística $(P>0.05)$ en el rendimiento de extracción de taninos, lo cual indica que no se apreciaron cambios en los niveles evaluados (Gráfica 2), por el contrario con el factor de concentración de $\mathrm{NaOH}$ (Gráfica 3) se observó que a una mayor alcalinidad la extracción fue mayor $(\mathrm{P}<0.05)$; estos resultados concuerdan con los de Chupin et al., (2013) quienes encontraron una relación directa entre la concentración del $\mathrm{NaOH}$ con el rendimiento de la extracción, 
la cual se incrementó cuando fueron mayores los niveles de alcalinidad en el extracto.

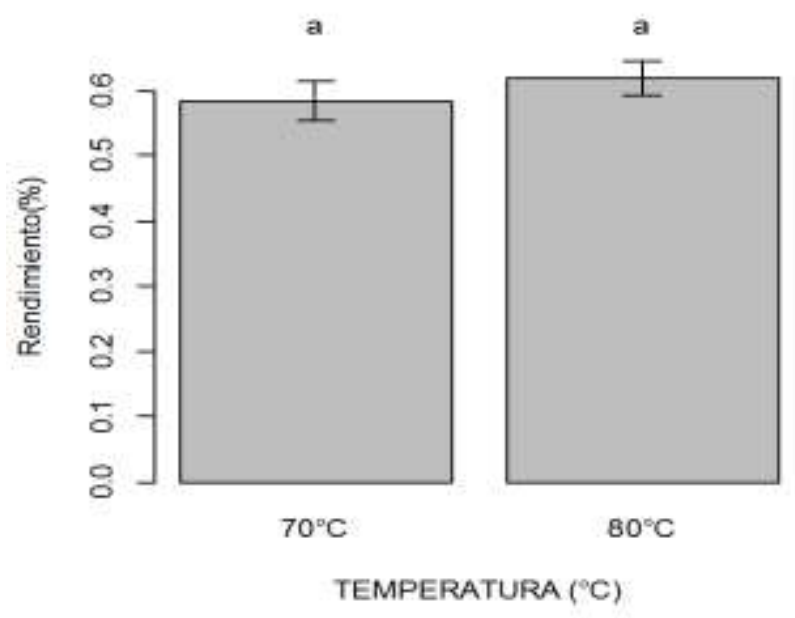

Figura 2. Porcentaje (\%) de rendimiento de extracción en función de la temperatura

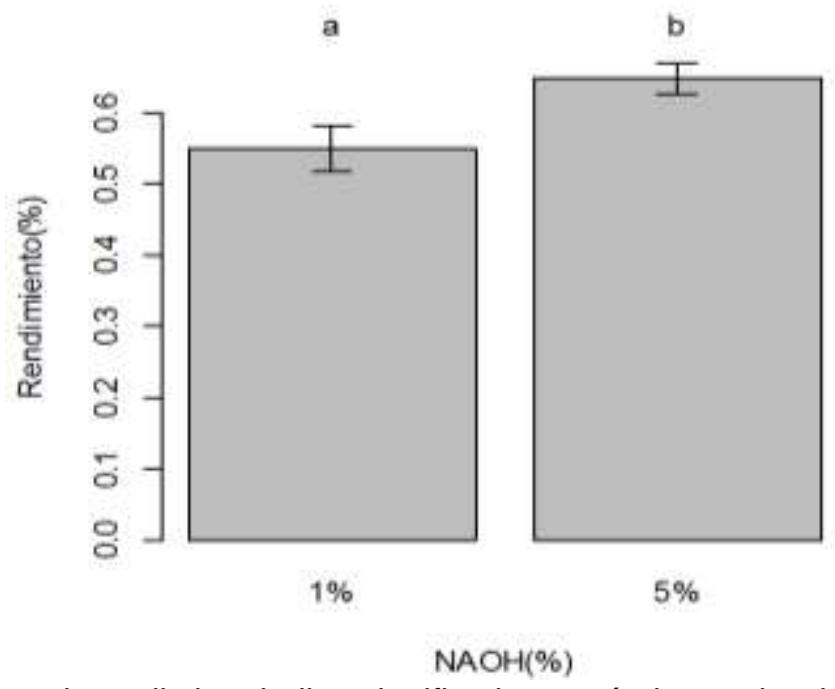

Promedios con letra distinta indica significativa según la prueba de Fisher $(\mathrm{P}<0.05)$

Figura 3. Porcentaje (\%) de rendimiento de extracción en función de la concentración de $\mathrm{NaOH}$ usada

La relación solido/líquido también influyó en el porcentaje de extracción, siendo la menor la de $1 / 60$, que obtuvo rendimientos del $0.55 \%$, mientras que la relación 1/90 
obtuvo la mayor extracción $(0.65 \%)(\mathrm{P}<0.05)$, aunque su comportamiento fue similar con la de 1/120 ( $P>0.05)$ (Gráfica 4). Estos resultados se explican porque la proporción 1/90 tiene un mejor equilibrio entre la cantidad de extracto y de solución, siendo en este caso el agua, sugiriendo que en la extracción de polifenoles no es conveniente ni un medio muy diluido, ni un medio muy saturado de corteza (Ping et al., 2011).

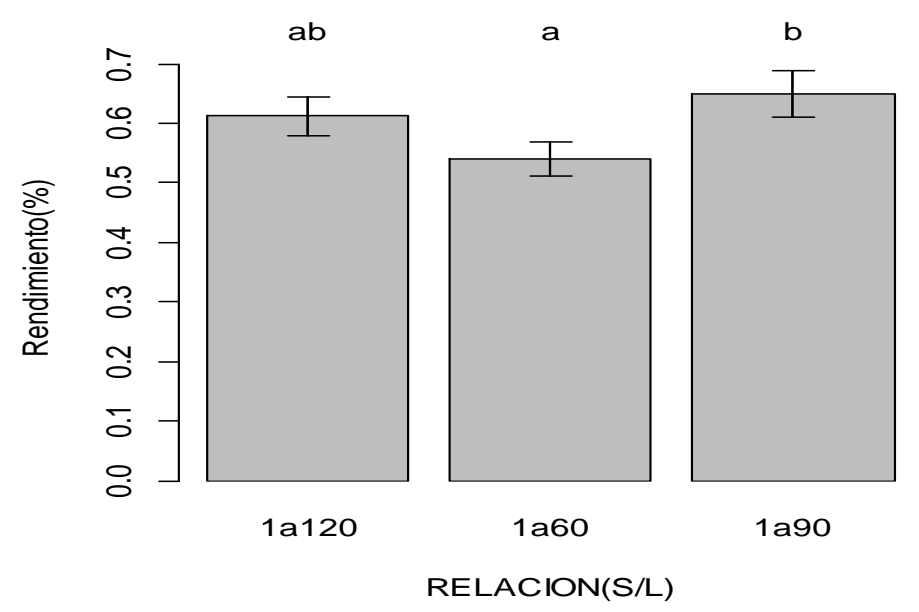

Promedios con letra distinta indican diferencias significativas según la prueba de Fisher $(P<0.05)$

Figura 4. Porcentaje (\%) de rendimiento de extracción en función de la relación sólido/líquido

Al combinar los diferentes factores evaluados (Gráfica 5), se puede observar que T11 y T3 fueron los tratamientos que generaron mayores rendimientos de extracción con valores de 0.85 y $0.80 \%$ respectivamente; sin embargo, T11 comprueba lo planteado anteriormente en relación a cada uno de los factores en función del rendimiento de extracción, por lo tanto, dicho tratamiento cumple con la composición ideal según esta investigación. Los tratamientos con rendimientos menores fueron T1 y T9, demostrando que la temperatura no es un factor de relevancia, y al mismo tiempo que la relación solido/líquido de 1/60 y 1/120, combinadas con una baja alcalinidad no genera buen rendimiento de extracción. Colín et al., (2013) afirman en su investigación que la mejor relación $S / L$ de extracción es 1/60, sin embargo, es conveniente una alcalinidad alta para mejorar dicho proceso. 


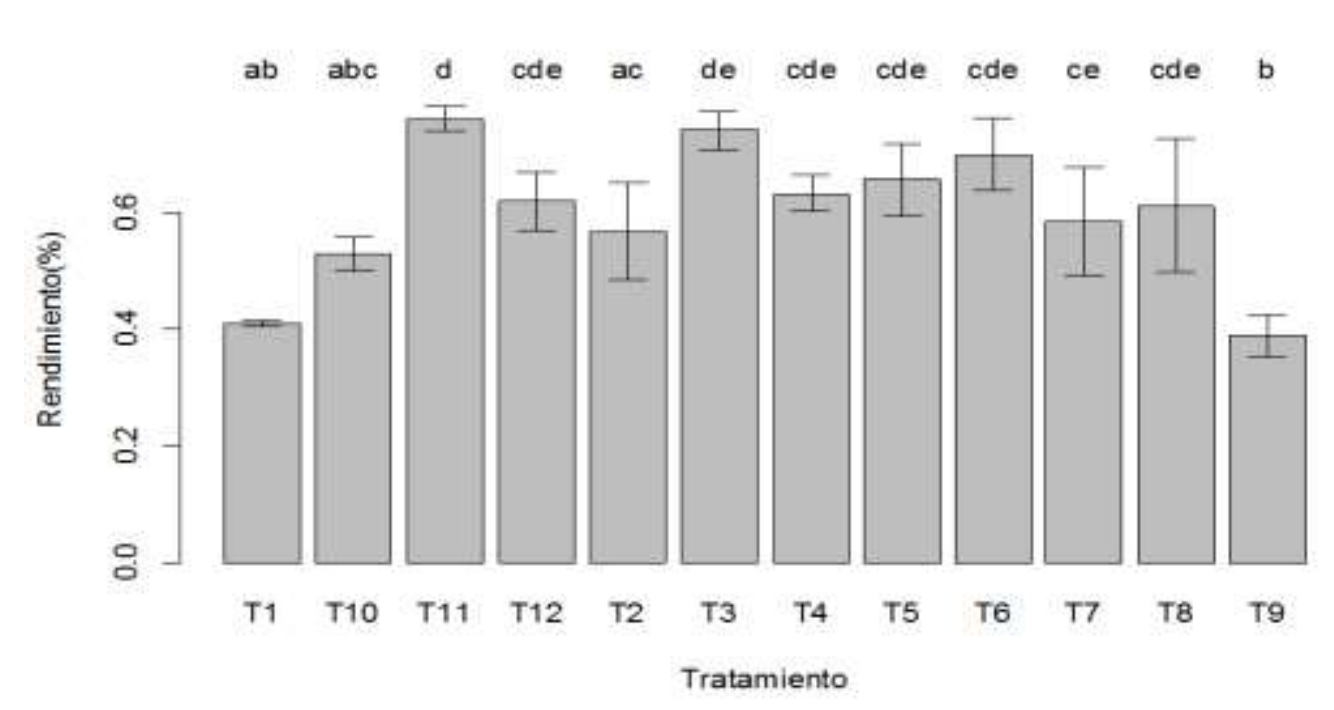

Promedios con letra distinta indica significancia según la prueba de Fisher $(P<0.05)$

Figura 5. Porcentaje (\%) de rendimiento de extracción de taninos en función del tratamiento

Para el caso de los árboles, el pino muestra mayor rendimiento con $\mathrm{NaOH}$ al $5 \%$, relación $\mathrm{S} / \mathrm{L}: 1 / 120$ y temperatura de $80^{\circ} \mathrm{C}$, siendo esta especie la de mayor porcentaje de extracción en comparación con las otras especies evaluadas, le sigue la acacia con $\mathrm{NaOH}$ al $1 \%, \mathrm{~S} / \mathrm{L}$ : $1 / 90$ y $80^{\circ} \mathrm{C}(\mathrm{P}<0.05)$, aunque se reitera que la temperatura no afecto el rendimiento extracción ( $P>0.05)$. El eucalipto mostró su mayor rendimiento con $\mathrm{NaOH}$ al $5 \%, \mathrm{~S} / \mathrm{L}: 1 / 90$ y $80^{\circ} \mathrm{C}$, aunque no se presentó diferencia $(P>0.05)$ en función de los factores que se analizaron (Figura 6). El porcentaje de $\mathrm{NaOH}$ con respecto a la relación $\mathrm{S} / \mathrm{L}$ tiene un comportamiento homogéneo en los dos niveles, mostrando mejores rendimientos con la relación 1/90 (Colín et al., 2013); y en la temperatura se presentó la misma situación puesto que no generó cambios importantes en el rendimiento de extracción $(P>0.05)$. Para el caso de la relación $S / L$ en función de la temperatura la interacción fue importante con $1 / 120$ a $80^{\circ} \mathrm{C}(\mathrm{P}<0.05)$, los demás tratamientos se comportaron sin variaciones $(P>0.05)$. Los resultados de esta investigación concuerda con lo reportado por dos Santos et al., (2017), estableciendo que la temperatura ideal de extracción está entre 60 y $80^{\circ} \mathrm{C}$, quizás ese fue el motivo por el cual este factor no alteró los resultados de forma significativa. 


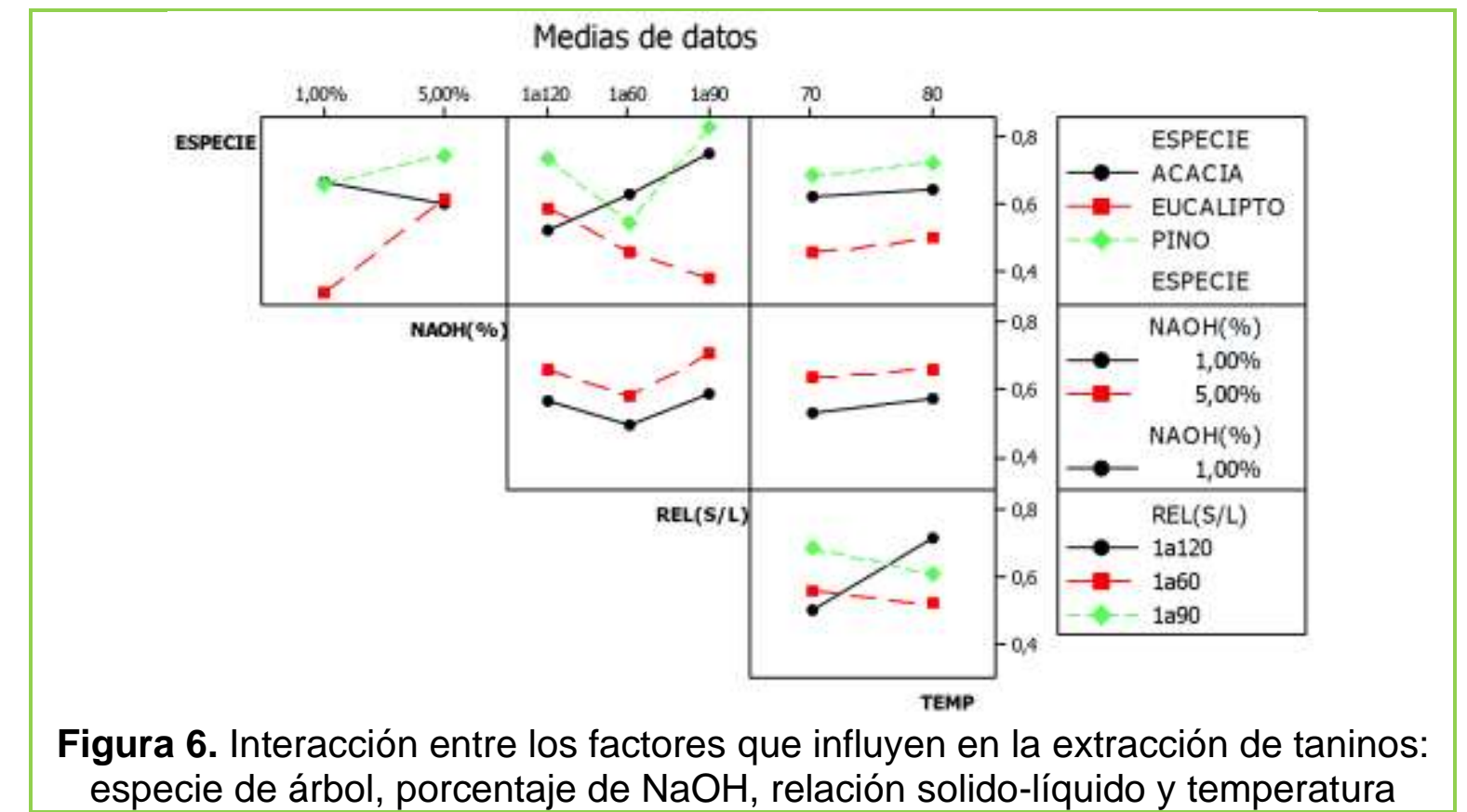

La corteza de pino presentó los mejores rendimientos con $5 \%$ de $\mathrm{NaOH}$ y relación $S / L$ de 1/90 puesto que la concentración de fenoles en este árbol se encuentra en una buena proporción con este extracto (Gráfica 7); mientras que en la Acacia mangium (Gráfica 8 ) aunque la relación $S / L$ siguió siendo la misma que generó buenos rendimientos en el pino, la mayor alcalinidad aplicada no funcionó, lo cual indica que indudablemente los niveles de sus metabolitos son diferentes a los del pino, lo cual influye en las condiciones de polaridad cuando reacciona con el $\mathrm{NaOH}$. Los taninos condensados tienen buena relación con la sal, los hidrolizables no tanto y pueden estar presentes en una buena concentración en la acacia (Filgueira et al., 2017), para un futuro estudio de caracterización puede determinarse si la acacia contiene taninos hidrolizables.

En el comportamiento del rendimiento de eucalipto es notable que la superficie de respuesta es diferente a las otras dos cortezas, su mayor extracción se presentó con la relación $S / L$ de $1 / 120$ y $5 \%$ de $\mathrm{NaOH}$, lo cual indica que estos factores influyeron en el contenido de taninos hidrolizables y en su afinidad con los reactivos (Lee et al., 2017). 


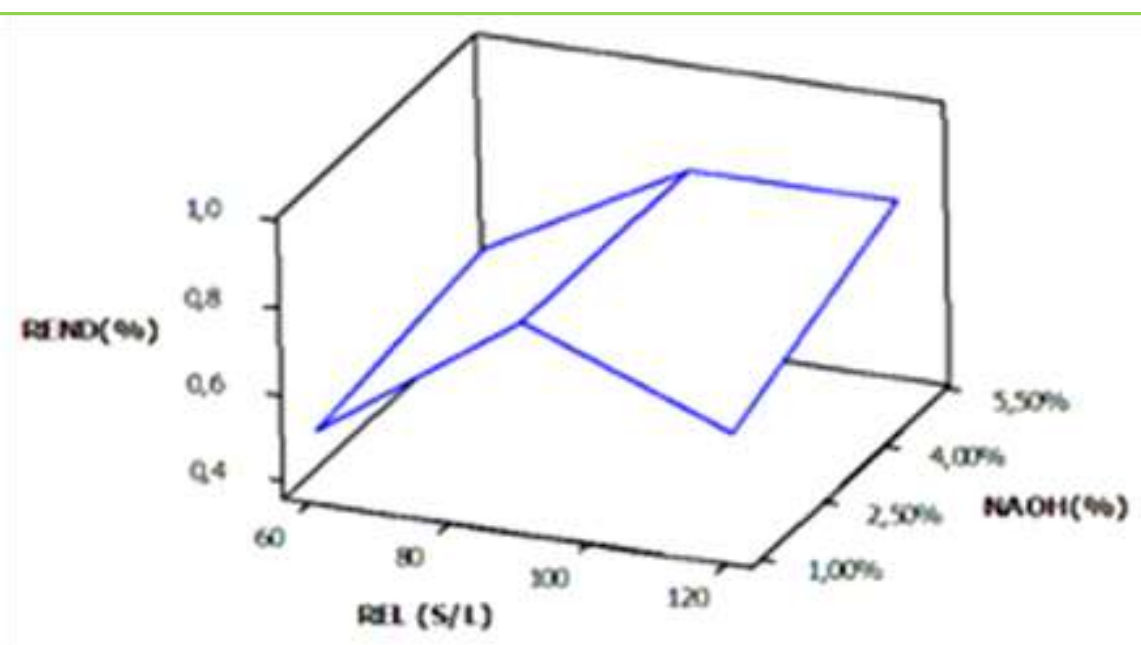

Figura 7. Superficie de respuesta del rendimiento (\%) de extracción de taninos en pino en función de la concentración de $\mathrm{NaOH}$ y relación solido/líquido (S/L)

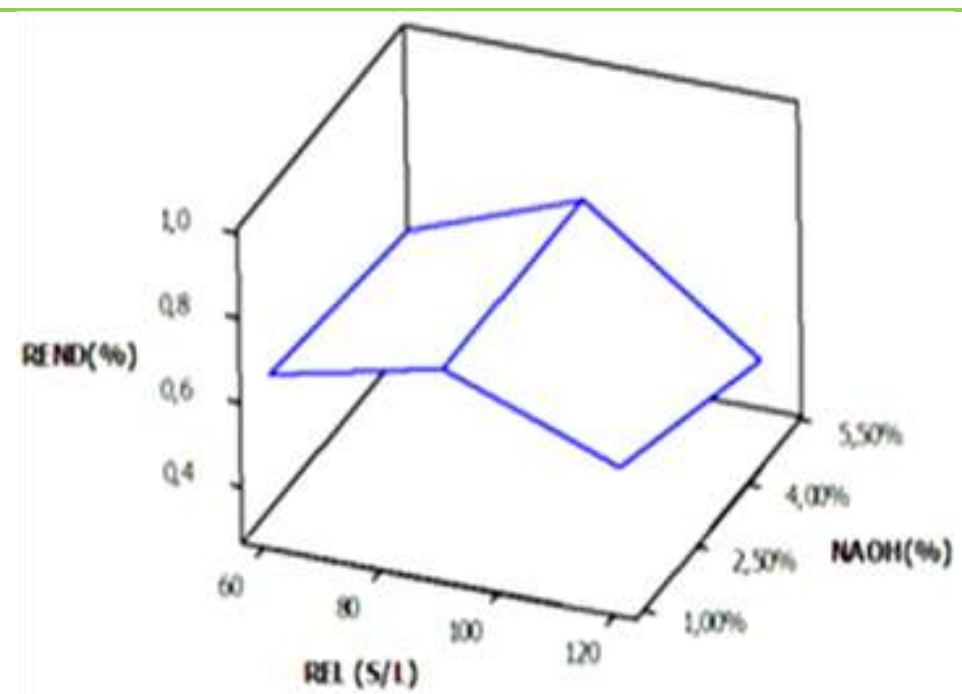

Figura 8. Superficie de respuesta del rendimiento (\%) de extracción de taninos de acacia en función de la concentración de $\mathrm{NaOH}$ y relación solido/líquido (S/L)

\section{CONCLUSIONES}

El mayor rendimiento de extracción de taninos que se observó en las tres especies de árboles según las condiciones de extracción para cada una fue: Pinus caribaea $\mathrm{NaOH}$ al $5 \%$, relación $\mathrm{S} / \mathrm{L} 1 / 90$ a temperatura de $80^{\circ} \mathrm{C}$; Eucalipto pellita de manera similar $\mathrm{NaOH} 5 \%$, pero con relación $\mathrm{S} / \mathrm{L} 1 / 120$ y también a $80^{\circ} \mathrm{C}$; y como último Acacia mangium $\mathrm{NaOH}$ al $1 \%, \mathrm{~S} / \mathrm{L} 1 / 90$ y $80^{\circ} \mathrm{C}$. Comparando las tres cortezas la de 
menor rendimiento fue eucalipto, mientras que la de pino fue la mayor, aunque su producción fue similar al de la acacia.

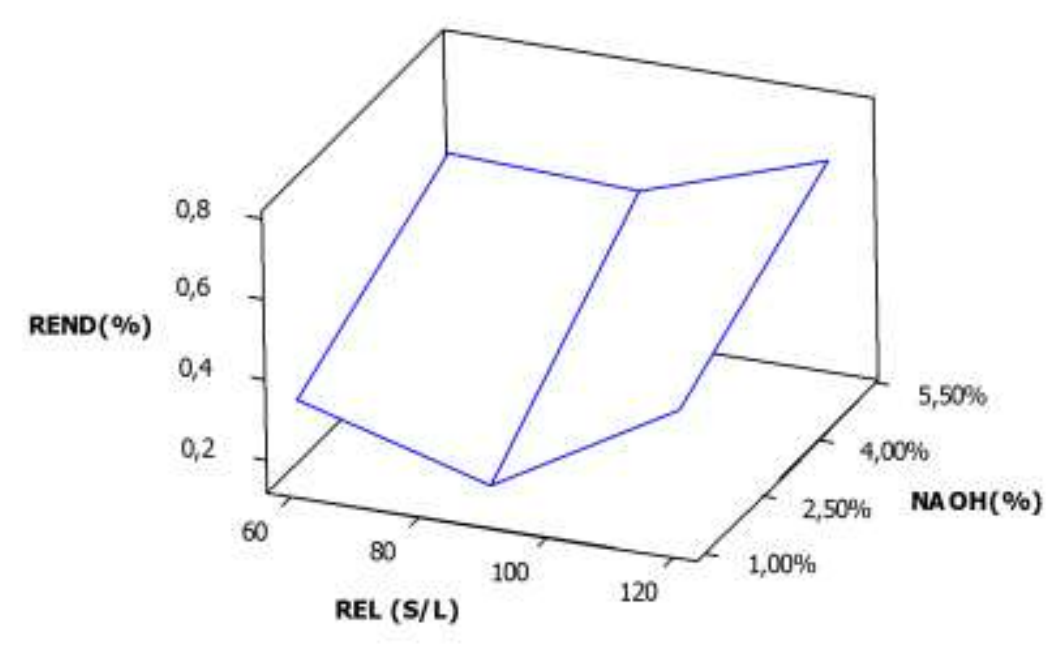

Figura 9. Superficie de respuesta del rendimiento (\%) de extracción de taninos en eucalipto en función de la concentración de $\mathrm{NaOH}$ y relación solido/líquido $(\mathrm{S} / \mathrm{L})$

Al evaluar el efecto de cada uno de los factores estudiados en el proceso de extracción de taninos, se observó que la temperatura no influyó probablemente debido que eran temperaturas muy cercanas; por el contrario, el factor de alcalinidad, es decir porcentaje de $\mathrm{NaOH}$, y la relación $\mathrm{S} / \mathrm{L}$ lograron una interacción en su mayoría directamente proporcional con el rendimiento de la extracción.

\section{REFERENCIAS BIBLIOGRÁFICAS}

1. Aguilar J., Jaén J.C., Vargas A.S., Jiménez P., Vega I., Herrera J., Borbón H., Soto R.M. Extracción y evaluación de taninos condensados a partir de la corteza de once especies maderables de Costa Rica. Revista Tecnología en Marcha. 25 (4): 15-22. 2012.

2. Álvarez J. Tanino. La revolución enológica mito o realidad. Revista Enología. 2 (IV): 1-15. 2007.

3. Amoako D.B., Awika J.M. Polymeric tannins significantly alter properties and in vitro digestibility of partially gelatinized intact starch granule. Food Chemistry. 208: 10-17. 2016.

4. Bernhard F., Main G., Holl K., Loumeto J., Ngao J. Fast disappearance of the water-soluble phenolic fraction in eucalypt leaf litter during laboratory and field experiments. Applied Soil Ecology. 23 (3): 273-278. 2003. 
5. Borralho N., Nieto V. Eucalyptus para la Orinoquia: retos y oportunidades, El mueble y la madera, 75: 26-33. 2012. Recuperado 10 Diciembre 2017. Disponible https://www.researchgate.net/publication/275892989 Eucalyptus para la Ori noquia retos y oportunidades

6. Casares A.B. Análisis de polifenoles en los vinos mediante técnicas de separación, Ingeniera Técnica Industrial Especialista en Química. Escola Universitaria d’Enginyeria Técnica Industrial de Barcelona, Universitat Politécnica de Catalunya, Barcelona. 70 p. 2010.

7. Colín S., Ochoa H.G., Rutiaga J.G. Contenido de taninos en la corteza de dos especies de parácata (Erythroxylon compactum Rose y Senna skinneri Benth. Irwin \& Barneby). Revista Chapingo serie ciencias forestales y del ambiente. 19 (1): 115-124. 2013.

8. Coronado M., Vega y León S., Gutiérrez R., Vázquez M., Radilla C. Antioxidantes: perspectiva actual para la salud humana. Revista Chilena de Nutrición. 42 (2): 206-212. 2015.

9. Chupin L., Motillon C., Charrier F., Pizzi A., Charrier B. Characterization of maritime pine (Pinus pinaster) bark tannins extracted under different conditions by spectroscopic methods, FTIR and HPLC. Industrial Crops and Products. 49: 897-903. 2013.

10. dos Santos C., Vargas Á., Fronza N., dos Santos J.H.Z. Structural, textural and morphological characteristics of tannins from Acacia mearnsii encapsulated using sol-gel methods: Applications as antimicrobial agents. Colloids and Surfaces B: Biointerfaces. 151: 26-33. 2017.

11. Durán T.M. Efecto de un deshoje tardío en la composición fenólica de semillas de bayas del cultivar Carménère, Ingeniera Agrónoma. Facultad de Ciencias Agronómicas, Universidad de Chile, Santiago, Chile. 38 p. 2010.

12. Filgueira D., Moldes D., Fuentealba C., García D. Condensed tannins from pine bark: A novel wood surface modifier assisted by laccase. Industrial Crops and Products. 103: 185-194. 2017.

13. Garrote G., Falqué E., Domínguez H., Parajó J.C. Autohydrolysis of agricultural residues: study of reaction byproducts. Bioresource Technology. 98 (10): 19511957. 2007.

14. Granados C., Yáñez X., Acevedo D. Evaluación de la actividad antioxidante del aceite esencial foliar de Myrcianthes leucoxyla de norte de Santander (Colombia). Información tecnológica. 25 (3): 11-16. 2014.

15. Jahanshahi S., Pizzi A., Abdulkhani A., Doosthoseini K., Shakeri A., Lagel M., Delmotte L. MALDI-TOF, $13 \mathrm{C}$ NMR and FT-MIR analysis and strength characterization of glycidyl ether tannin epoxy resins. Industrial Crops and Products. 83: 177-185. 2016.

16. Jerez M., Pinelo M., Sineiro J., Núñez M.J. Influence of extraction conditions on phenolic yields from pine bark: assessment of procyanidins polymerization degree by thiolysis. Food chemistry. 94 (3): 406-414. 2006.

17. Lee D.Y., Kim H.W., Yang H., Sung S.H. Hydrolysable tannins from the fruits of Terminalia chebula Retz and their a-glucosidase inhibitory activities. Phytochemistry. 137: 109-116. 2017 
18. Min K., Freeman C., Kang H., Choi S.-U. The regulation by phenolic compounds of soil organic matter dynamics under a changing environment. BioMed research international. Article ID 825098: 1-11. 2015.

19. Nadhari W.N.A.W., Hashim R., Hiziroglu S., Sulaiman O., Boon J.G., Salleh K.M., Awalludin M.F., Sato M., Sugimoto T. Measurement of some properties of binderless composites manufactured from oil palm trunks and Acacia mangium. Measurement. 50: 250-254. 2014.

20. Naima R., Oumam M., Hannache H., Sesbou A., Charrier B., Pizzi A., Charrier F. Comparison of the impact of different extraction methods on polyphenols yields and tannins extracted from Moroccan Acacia mollissima barks. Industrial Crops and Products. 70: 245-252. 2015.

21. Olivas F.J., Wall A., González G.A., López J.A., Álvarez E., Rosa L.A., Ramos A. Taninos hidrolizables: bioquímica, aspectos nutricionales y analíticos y efectos en la salud. Nutrición Hospitalaria. 31 (1): 55-66. 2015.

22. Ping L., Pizzi A., Guo Z.D., Brosse N. Condensed tannins extraction from grape pomace: characterization and utilization as wood adhesives for wood particleboard. Industrial Crops and Products. 34 (1): 907-914. 2011.

23. Promoción de Turismo, Inversión y Exportaciones (PROEXPORT). Sector Forestal en Colombia. Bogotá, Colombia. 17 p. 2012.

24. Quiñones M., Miguel M., Aleixandre A. Los polifenoles, compuestos de origen natural con efectos saludables sobre el sistema cardiovascular. Nutrición Hospitalaria. 27 (1): 76-89. 2012.

25. Villanueva J., Cerano J., Fulé P.Z., Cortés C., Vázquez L., Yocom L.L., Ruizl J.A. Cuatro siglos de variabilidad hidroclimática en el noroeste de Chihuahua, México, reconstruida con anillos de árboles. Investigaciones Geográficas. 87: 141-153. 2015.

26. Wadnerkar D., Tade M.O., Pareek V.K., Utikar R.P. CFD simulation of solidliquid stirred tanks for low to dense solid loading systems. Particuology. 29: 1633. 2016. 